\title{
ICD-11 Trauma Questionnaires for PTSD and Complex PTSD: Validation among Civilians and Former Abducted Children in Northern Uganda
}

\section{Sarah Dokkedah', Henry Oboke ${ }^{2}$, Emilio Ovuga ${ }^{3}$ and Ask Elklit ${ }^{1 *}$}

${ }^{1}$ National Center of Psychotraumatology, Department of Psychology, University of Southern Denmark

${ }^{2}$ Department of Mental Health, Gulu University, Uganda

${ }^{3}$ Faculty of Medicine, Gulu University, Uganda

\begin{abstract}
Objectives: ICD-11 is expected to introduce a new diagnosis of C-PTSD, along with a revision of the current PTSD diagnosis. Are the suggested diagnostic tools for PTSD and C-PTSD valid in a developing country?

Method: The tools have been tested on former abducted and regular civilians in northern Uganda $(n=314)$, who have been influenced by the civil war that lasted for more than two decades.

Results: The prevalence of either PTSD or C-PTSD was $36.6 \%$ and PTSD and C-PTSD was further found to correlate with symptoms of depression, anxiety and somatic complaints.

Conclusion: Based on its findings the study concludes that the ICD-11 tools for PTSD and C-PTSD both appear to be valid as suggested by both discriminant and convergent validation of the tools. However, future research can benefit from studying cultural aspects of these diagnoses.
\end{abstract}

\section{Keywords: PTSD; C-PTSD; War; Abduction; Children; ICD-11}

\section{Introduction}

The current paper focuses on disorders specifically associated with stress, particularly 'post-traumatic stress disorder' (PTSD) and 'complex PTSD' (C-PTSD) in northern Uganda. Judith Herman was the first to introduce the concept of C-PTSD, in 1992 [1]. Herman reviewed the evidence of a complex form of PTSD in survivors of prolonged, repeated trauma. Herman argued that clinical observations could identify three broad areas of disturbance that transcend PTSD; a) the symptom picture often appears to be more complex, diffuse, and persisting, b) the development of characteristic personality changes, and c) a vulnerability to repeated harm towards self and others. C-PTSD was described as coexisting with 'simple' PTSD, but extending beyond it [1]. Disorders specifically associated with stress are among the most widely used diagnoses amongst psychologists and psychiatrists worldwide. PTSD is the eighth most frequently used diagnosis for psychologists who used ICD-10 and the 14th most frequently used diagnosis for psychiatrists (not including mental retardation). For psychologists who use the DSM-IV, PTSD is the third most frequently used diagnosis [2,3]. The preparations of the fifth edition of the Diagnostic and Statistical Manual of Mental Disorders (DSM-5) and the 11th edition of the International Classification of Diseases and Related Mental Health Problems (ICD-11) have been overlapping. The DSM-5 was released in 2013, while the ICD-11 is expected to be released in 2017. However, the proposal of the ICD-11, which is already known, reveals significant differences between the two diagnostic systems [4].

For the ICD-11, the WHO appointed a Working Group, chaired by Andreas Maercker, to examine and improve the classification of a mixed group of conditions, including both 'reactions to severe stress and adjustment disorders' (ICD-10 code F43) and 'enduring personality change after catastrophic experiences' (ICD-10 code F62.0) [4]. In the new editions of the ICD and DSM, both manuals have implemented a separate grouping for disorders related to stress and are therefore no longer combining them with anxiety disorders. Both diagnostic systems include PTSD and 'adjustment disorder' as a part of this grouping $[4,5]$. For the DSM-5, the biggest change is the exclusion of the A2 criteria (i.e. the necessity of an intense trauma response of fear, helplessness, or horror). However, the DSM-5 is made as a "living document" that can be revised, as new research findings are published [6]. The DSM-5 has not included C-PTSD as a diagnosis and the tentative diagnosis of 'disorder of extreme stress not otherwise specified' (DESNOS) has been excluded. Friedman [6] argues that this diagnosis was excluded due to the minimal research on the topic since the DSM-IV. However, changes in the DSM-5 provide the opportunity to consider some core elements of C-PTSD with a number of symptoms (e.g. the new D cluster for negative cognitions and mood) in addition to the dissociative subtype that provides a specific diagnostic niche for individuals who have severe PTSD. Due to these additional symptoms the PTSD criteria for DSM-5 has been expanded from 17 symptoms to 20 symptoms and thereby extends from the proposed ICD-11 diagnosis, which contains seven symptoms based on the three core elements of PTSD $[4,6]$.

According to the ICD-11, PTSD is a disorder that develops following exposure to an "extremely threatening or horrific event or series of events" (4 p. 200, 1l. 3-4), while C-PTSD is a disorder that arises after "exposure to a stressor typically of an extreme or prolonged nature and from which escape is difficult or impossible" (p. 200, 11. 12-13). PTSD and C-PTSD can be said to be "sibling" disorders in a scenario where the category of traumatic stress disorders is the "parent". The stressor acts as to open a "gate" with the possibility of either a

*Corresponding author: Ask Elklit, National Center of Psychotraumatology Department of Psychology, University of Southern Denmark, Tel: +456550 2320; E-mail: aelklit@health.sdu.dk

Received September 25, 2015; Accepted October 23, 2015; Published October 30, 2015

Citation: Dokkedahl S, Oboke H, Ovuga E, Elklit A (2015) ICD-11 Trauma Questionnaires for PTSD and Complex PTSD: Validation among Civilians and Former Abducted Children in Northern Uganda. J Psychiatry 18: 335 doi:10.4172/2378-5756.1000335

Copyright: @ 2015 Dokkedahl S, et al. This is an open-access article distributed under the terms of the Creative Commons Attribution License, which permits unrestricted use, distribution, and reproduction in any medium, provided the original author and source are credited 
PTSD or a C-PTSD diagnosis, which is determined by the symptom profile. This allows the clinician to focus on symptoms, problems, and the target of treatment rather than the trauma history itself [7]. The current PTSD diagnosis has been criticized for its high comorbidity with other diagnoses and for the composition of its symptom clusters, which are believed to be too broad. Initial critique came from Simms et al., [8] who studied posttraumatic stress symptoms (PTSS) in veterans of the Gulf War and suggested that four factors underlie PTSD symptomatology; intrusions or re-experiencing symptoms, avoidance of traumatic stimuli, dysphoria or general distress and hyperarousal. Of these, they explained that dysphoria might more accurately be specified as a nonspecific general distress/negative affectivity component, which is similar in many other disorders. Based on their findings, Simms et al. [8], recommended refocusing the diagnostic assessment of PTSD to decrease comorbidity with other anxiety and depressive disorders. Specific critique directed against the DSM-IV pertains to be the more than 10,000 different combinations that exist based on the 17 different symptoms that can result in a PTSD diagnosis [4,9]. Likewise, studies suggest that the ICD-10 diagnosis has a relatively low threshold for a PTSD diagnosis [10]. The aim of the redefinition of PTSD in ICD-11 is to differentiate PTSD from normal reactions to extreme stressors and to reduce the comorbidity with other disorders by identifying the core elements of PTSD rather than the typical features of the disorder. The WHO Working Group for ICD-11 have identified three core elements in the PTSD disorder similar to DSM-IV: (1) re-experiencing the traumatic event(s) in the present; (2) avoidance of these intrusions; and (3) an excessive sense of current threat. The expectation is that these changes will help simplify the diagnosis and thereby direct the attention of the clinicians to the co-occurrence of the three core elements, of which all should be present for a diagnosis [4].

C-PTSD is a new disorder category that typically follows severe stressors over an extended period of time or multiple or repeated negative events from which separation is not possible (e.g. abduction or child soldiering). In addition to the three core elements of PTSD, C-PTSD consists of disturbances in the domains of affect, self-concept and relational functioning or a tendency towards experiencing prolonged dissociative states when under stress. Problems in the domain of affect can include difficulties in emotional regulation of both hyperactivation and deactivation, but it can also manifest itself as behavioral disturbances like violent outbursts and reckless or selfdestructive behavior. Problems with self-concept result in negative beliefs about oneself as diminished, defeated, or worthless. Feelings of shame, guilt, or failure can furthermore be related to the self-concept. Disturbances in relational functioning are known to be associated with difficulties with feeling close to others. This is reflected in the constant avoiding, deriding, and lack of interest in personal relationships and general social engagements [4]. In a latent profile analysis, Cloitre et al. [7] found support for the proposed ICD-11 distinction between PTSD and C-PTSD. The study found that individuals with C-PTSD experienced greater functional impairment than individuals with PTSD. The study further showed that different symptom profiles describing PTSD and C-PTSD are associated with different subgroups of individuals, different levels of impairment, and different traumatic events. With the inclusion of three large clinical samples, Elklit et al. [11] conducted a similar study that provided support for the diagnostic structure of C-PTSD. The study indicated that C-PTSD is associated with a broad range of psychological problems.

\section{Uganda}

From 1986-2007 Uganda was affected by the civil war between the government and the Lords Resistance Army (LRA). During the period of the conflict in northern Uganda, an estimated 25.000 children were abducted for the use of child soldiers by the LRA and many civilians fled to camps for internally displaced persons (IDPs) [12]. Numerous studies have documented severe mental health problems in northern Uganda. Pfeiffer and Elbert [13] studied former abductees and found that $49 \%$ of their participants could be diagnosed with PTSD, while $71 \%$ presented symptoms of depression and $60 \%$ presented symptoms of anxiety. Thirty-six percent were found to fulfill the criteria for PTSD, depression, and anxiety simultaneously [13]. A longitudinal study, the War-Affected Youth Study (WAYS), by Amone-P'Olak et al. [14], found that war experiences were significantly associated with difficulties performing daily activities and tasks $(\mathrm{t}=-2.62, \mathrm{p}<.001)$, especially for females compared to male participants $(\mathrm{t}=-2.62, \mathrm{p}<.05)$. Overall, women were more at risk of long-term mental health outcomes compared to men [14]. Due to the extent of trauma found in previous studies and the prolonged period of the war, we presuppose that northern Uganda has a relevant population for the study of C-PTSD. The following study includes both former abducted children and never abducted civilians of the Awach community in the Gulu district. The study uses the proposed measurement tools for the ICD-11 PTSD and C-PTSD diagnosis respectively [15]. The aim of this study was to examine the degree to which the new diagnostic proposal of ICD-11 is suited to describe the consequences of traumatic experiences in a developing country. These diagnoses are expected to be more simple and easier to use in low-resource and humanitarian settings as stated by Maercker et al. [5]. We expect that potential traumatic experiences from the conflict in northern Uganda will have high associations with PTSD and C-PTSD. We further expect that childhood traumas and long-lasting traumatization deriving from either war traumatization or childhood abuse will be correlated with high PTSD as well as C-PTSD scores.

\section{Methods}

\section{Sample}

This cross-sectional study was conducted in Gulu, in the subcounty of Awach, the biggest district of northern Uganda. The sample $(\mathrm{N}=314)$ included both males (49\%) and females (51\%) in the age group 18-25 years (Table 1). This age group was still children when the war was ongoing. Forty percent (39.5\%) of the participants included were former abductees (FA) from the LRA $(\mathrm{N}=124)$ while the remaining participants were never abducted (NA) civilians $(\mathrm{N}=190)$. The lack of a national register in Uganda made random sampling difficult. The local leaders (LC1's) of the four parishes in Awach therefore selected the participants based on the principles of random sampling. The LC1's included the number of participants from each parish in accordance to the size of the parish. Participants were further included equally from each village within the parish with equal participation between genders, age and FA vs. NA. This allowed for a broad distribution of participants within the Awach community. The LC1's were asked to exclude any participants with known psychotic symptoms or mental retardation who would be unable to complete the interview. Participants gave written consent to their participation and those who could not write were asked to sign with their thumbprint in ink. Before giving consent the participants were informed about the content of the study, their rights to decline and withdraw at any time, and the confidentiality of their participation in the study. None of the participants who were selected declined to participate. The questionnaires were read out loud for the participants to avoid any possible reading disabilities in the rural 
Citation: Dokkedahl S, Oboke H, Ovuga E, Elklit A (2015) ICD-11 Trauma Questionnaires for PTSD and Complex PTSD: Validation among Civilians and Former Abducted Children in Northern Uganda. J Psychiatry 18: 335 doi:10.4172/2378-5756.1000335

Page 3 of 9

areas of northern Uganda. Local field assistants asked the questions in the local language, Luo, which is the language spoken by the Acholi tribe. The interviews took place at the homes of the participants at a quiet spot, allowing for breaks if needed.

\section{Measures/instruments}

All measures and instruments were translated and back-translated from English into Luo the local language of the Awach community. The Institutional Review and Ethics Committee at the Lacor Hospital in Gulu approved the project along with the translations. The participants were asked about demographic characteristics. In addition to questions about age and gender, the participants were asked about their parents education, their living arrangements, how many of five assets their household owned (bicycle, running water, electricity, car, and television), how many people were sleeping in the same room, and the number of meals a day. Furthermore, the participants were asked about their tribe and if they had ever been abducted by the LRA. If yes, the participants were asked about their age at abduction and the duration of their abduction.

\section{Trauma checklist}

The trauma exposures of the participants were measured using the UNICEF War Trauma Screening Scale, [16] which was originally developed for Bosnia. The adapted version for West African war-affected youth was used, which had been altered by Amone-P'Olak et al. [14] to better capture the context of war in African countries (Table 2).

\begin{tabular}{|c|c|c|c|c|}
\hline \multicolumn{5}{|l|}{ Means (SD) of the Sample } \\
\hline & $\begin{array}{l}\text { Former Abducted } \\
\qquad(\mathrm{N}=22)\end{array}$ & $\begin{array}{l}\text { Never Abducted } \\
\quad(\mathrm{N}=189)\end{array}$ & $\begin{array}{l}\text { Total sample } \\
(\mathrm{N}=314)\end{array}$ & Statistics \\
\hline Age & $M=22.8(2.51)$ & $\mathrm{M}=22.11(2.55)$ & $\mathrm{M}=22.4(2.6)$ & $\mathrm{F}=4.80 \mathrm{P}<.05$ \\
\hline Sex \% & $\begin{array}{c}\text { Female: } 47.6 \\
\text { Male: } 51.6\end{array}$ & $\begin{array}{c}\text { Female: } 53.4 \\
\text { Male: } 46.6\end{array}$ & $\begin{array}{l}\text { Female:51 } \\
\text { Male: } 48.7\end{array}$ & $\begin{array}{c}\mathrm{F}=.89 \\
\mathrm{~ns}\end{array}$ \\
\hline Owned Assets & $M=0.7(0.66)$ & $M=0.73(0.69)$ & $M=0.72(0.68)$ & $\begin{array}{c}\mathrm{F}=.20 \\
\mathrm{~ns}\end{array}$ \\
\hline Meals a Day & $M=1.9(0.47)$ & $M=2.03(0.59)$ & $M=1.98(0.55)$ & $F=4.56 P<.05$ \\
\hline Number of People Sleeping in the Same Room & $M=4.12(1.73)$ & $M=3.7(1.52)$ & $M=3.86(1.6)$ & $F=5.03 P<.05$ \\
\hline Fathers Education ${ }^{A}$ & $M=1.95(.65)$ & $M=2.15(.74)$ & $M=2.07(.71)$ & $\mathrm{F}=5.87 \mathrm{P}<.02$ \\
\hline Mothers Education ${ }^{A}$ & $M=1.58(.62)$ & $M=1.66(.63)$ & $M=1.63(.63)$ & $\mathrm{F}=1.23 \mathrm{~ns}$ \\
\hline \multicolumn{5}{|l|}{ Living with \%: } \\
\hline ‘One parent’ & 42.4 & 37.1 & 39.0 & \\
\hline 'Both parents' & 33.9 & 43.0 & 39.7 & $\mathrm{~F}=1.17$ \\
\hline 'Husband/wife' & 12.7 & 12.9 & 12.8 & $\mathrm{~ns}$ \\
\hline 'Other’ & 11.0 & 6.9 & 8.5 & \\
\hline
\end{tabular}

AThe parents education was scored as 1='none', 2='Primary School', 3='Secondary School', 4='Diploma', 5='Bachelor Degree' and 6 ='Masters Degree'. ns=non-significant Table 1: Demographic statistics.

\section{Percent Endorsement Trauma Exposure Checklist}

\section{A. Personal Injury}

1. Did you get injured during the war?

2. Did you feel threatened during the war and thought that you may die?

3. Were you arrested and imprisoned during the war?

4. Were you beaten, slapped or tortured during the war?

5. Were you injured intentionally by someone during the war?

6. During the war, were you denied eating food or water for a long time so you thought you might die?

B. Witnessing violence

7. During the war, did you see somebody getting killed or injured badly?

8. During the war, did you see a dead body?

9. During the war, did you see anybody getting seriously injured?

10. During the war, did you touch or carry an injured or dead body?

11. Did you see anybody getting beaten during the war?

12. Did you see anybody getting injured during the war?

13. During the war, did you see anyone getting arrested?

14. During the war, did you ever see anybody getting confused after hearing about the death of his or her dear ones?

15. During the war, did you see anybody getting killed, beaten, injured, or arrested?

16. During the war, did you see activities like bombardments of bridges, arsons or breaking bridges?

17. During the war, did you see somebody being killed?

C. Injuries and threats to self or loved ones

18. Have you experienced bullets passing near you and almost getting hit?

19. Did you ever experience land mines or bombs landing near you and coming close to injuring you?

20. During the war, did either rebels or government soldiers forcefully enter your house?

Former

Abducted

Never Abducted Total Sample

\begin{tabular}{|l|l|l|}
\hline 44.4 & 12.2 & 24.8 \\
\hline 93.5 & 45.5 & 64.3 \\
\hline 77.4 & 14.3 & 39.5 \\
\hline 90.3 & 31.7 & 55.1 \\
\hline 64.5 & 17.5 & 36.0 \\
\hline 90.3 & 20.6 & 48.1 \\
\hline \multicolumn{3}{|c|}{} \\
\hline 90.3 & 55.0 & 69.1 \\
\hline 95.2 & 81.0 & 86.6 \\
\hline 89.5 & 68.8 & 77.1 \\
\hline 69.4 & 28.0 & 44.6 \\
\hline 91.1 & 67.7 & 76.8 \\
\hline 89.5 & 61.9 & 72.6 \\
\hline 85.5 & 48.1 & 63.1 \\
\hline 61.3 & 49.2 & 54.1 \\
\hline 85.5 & 60.8 & 70.4 \\
\hline 91.1 & 75.7 & 81.8 \\
\hline 89.5 & 59.8 & 71.3 \\
\hline 92.7 & 70.4 & 79.3 \\
\hline 74.2 & 40.2 & 53.8 \\
\hline 91.1 & 78.3 & 83.4 \\
\hline
\end{tabular}


Citation: Dokkedahl S, Oboke H, Ovuga E, Elklit A (2015) ICD-11 Trauma Questionnaires for PTSD and Complex PTSD: Validation among Civilians and Former Abducted Children in Northern Uganda. J Psychiatry 18: 335 doi:10.4172/2378-5756.1000335

Page 4 of 9

21. Were you threatened with death or injury?

22. In any single day, during the war, did you ever think somebody could kill you?

D. Deaths

23. Was your father killed during the war?

24. Was your mother killed during the war?

25. Did your loved ones kill him/herself during the war?

26. Was your sister or brother killed during the war?

27. Was your close relative killed during the war?

28. Was your close friend killed during the war?

29. Did your close friend/loved ones die of cancer or heart disease during the war?

E. Threats to loved ones

30. Did any close friend/loved ones get beaten during the war?

31. During the war, did anybody you love get injured?

32. During the war, was anybody you love tortured?

33. During the war, were there people you love who was arrested as prisoners?

F. Material losses

34. Did your home(s) get destroyed during the war?

35. Were you displaced because of the war?

36. Were you displaced out of your village, during the war?

37. Did you leave your valuable items as a result of the war?

G. Threats to your loved ones

38. During the war, did anyone you love lead atrocities against civilian?

39. During the war, was there anybody you love who died of cancer or heart disease/attack?

40. During the war, were there cases of missing persons of which you to this date do not know about his/her whereabouts and if he/she is alive or dead

41. During the war, was there anybody who expressed an idea of killing your loved ones?

H. Separation

42. During the war, were you separated from your love ones?

43. During the war, were you separated from your parents?

I. Displacement

44. Did you run and leave your home(s) as a result of the war?

45. Did you leave your home, district or town?

46. Have you changed your school or work station during the war?

47. Did you stay in IDP camp?

48a. Did you leave your country because of the war?

J. Participating in armed groups

49a. Did you fight for any armed group?

50a. Were you trained in military tactics?

50b. After the war, did you receive counseling from bodies like GUSCO, World Vision, KICWA?

50c. During the war, were you forced to kill, drink blood, smoke marijuana or something that might have changed your mind?

K. Sexual abuse

51a. Sometimes during the war, do you remember somebody being raped?

$51 \mathrm{~b}$. Did you see any family members being raped?

51c. Were you sexually abused?

Total of Experiences

Table 2: Traumatic experiences.

\begin{tabular}{|c|c|c|}
\hline 94.4 & 58.2 & 72.6 \\
994.4 & 72.0 & 80.6 \\
\hline \multicolumn{3}{|c|}{} \\
\hline 21.0 & 18.5 & 19.4 \\
\hline 12.9 & 5.8 & 8.6 \\
\hline 37.1 & 23.3 & 28.7 \\
\hline 54.8 & 42.9 & 47.8 \\
\hline 91.9 & 85.2 & 87.9 \\
\hline 78.2 & 63.5 & 69.1 \\
\hline 58.1 & 44.4 & 49.7 \\
\hline
\end{tabular}

\begin{tabular}{|l|l|l|}
\hline 96.8 & 81.5 & 87.6 \\
\hline 95.2 & 84.1 & 88.5 \\
\hline 92.7 & 84.7 & 87.9 \\
\hline 91.1 & 75.1 & 81.5 \\
\hline
\end{tabular}

\begin{tabular}{|l|l|l|}
\hline 100 & 94.2 & 96.5 \\
\hline 99.2 & 95.8 & 97.1 \\
\hline 99.2 & 94.7 & 96.5 \\
\hline 99.2 & 93.1 & 95.5 \\
\hline
\end{tabular}

\begin{tabular}{|l|l|l|}
\hline 71.0 & 67.2 & 68.5 \\
\hline 44.4 & 39.7 & 41.4
\end{tabular}

\begin{tabular}{l|l|r}
71.0 & 67.2 & 68.5 \\
\hline 44.4 & 39.7 & 41.4
\end{tabular}

\begin{tabular}{|l|l|l|}
\hline 41.4 & 39.7 & 41.4 \\
\hline 81.5 & 72.5 & 75.8 \\
\hline
\end{tabular}

\begin{tabular}{|l|l|r}
\hline 80.6 & 62.4 & 69.4 \\
\hline
\end{tabular}

\begin{tabular}{l|l|r}
96.8 & 91.0 & 93.3 \\
\hline
\end{tabular}

\begin{tabular}{|l|l|r}
\hline 98.4 & 91.0 & 93.9 \\
\hline
\end{tabular}

\begin{tabular}{l|l|r}
99.2 & 95.2 & 96.8 \\
\hline
\end{tabular}

\begin{tabular}{l|l|r}
93.5 & 95.8 & 94.9
\end{tabular}

\begin{tabular}{l|l|r}
\hline 92.7 & 93.7 & 93.0 \\
\hline
\end{tabular}

\begin{tabular}{l|l|r}
\hline 94.4 & 92.1 & 92.7 \\
\hline
\end{tabular}

\begin{tabular}{l|l|l}
\hline 4.0 & 2.6 & 3.2 \\
\hline
\end{tabular}

\begin{tabular}{|c|c|c|}
\hline 61.3 & 1.6 & 25.2 \\
\hline 54.8 & 1.1 & 22.3 \\
\hline 37.9 & .5 & 15.3 \\
\hline 41.9 & 7.9 & 21.3 \\
\hline
\end{tabular}

\begin{tabular}{|c|c|c|}
\hline 62.9 & 24.9 & 39.8 \\
\hline 65.3 & 16.9 & 36.0 \\
\hline 20.2 & 1.6 & 8.9 \\
\hline $\mathbf{4 2 . 8 6}$ & $\mathbf{3 0 . 4 1}$ & $\mathbf{3 4 . 6 6}$ \\
\hline
\end{tabular}

\section{ICD-11}

The ICD-11 Trauma Questionnaires were developed in 2013 by Cloitre, Roberts, Bisson, and Brewin [15]. They consist of two diagnostic questionnaires for PTSD and C-PTSD respectively. The questionnaire for PTSD contains seven questions with three clusters based on the core elements of PTSD: Re-experiencing the traumatic event, avoidance, and excessive sense of current threat (hypervigiliance). Each cluster needs at least one symptom score $>2$ to fulfill the PTSD diagnosis [15]. The questionnaire for C-PTSD contains 17 questions with four scoring clusters. Two clusters regard emotional regulation of either hyperactivation or deactivation, one cluster regards negative self-concept, and the last cluster regards disturbed relationships. Each cluster has a different threshold, which needs to be fulfilled to receive the diagnosis of C-PTSD. Both scales are measured on a 5 point Likert scale of $0=$ 'not at all', $1=$ 'a little bit', $2=$ 'moderately', $3=$ ='quite a bit', $4=$ 'extremely'[15].

\section{African youth psychosocial assessment}

Mental health was furthermore assessed by the African Youth Psychosocial Assessment Instrument (APAI), which is a field-based instrument developed for use in northern Uganda [14].

\section{The mini international neuropsychiatric interview}

The Mini International Neuropsychiatric Interview (M.I.N.I) is a brief structured interview designed for major Axis I psychiatric 
Citation: Dokkedahl S, Oboke H, Ovuga E, Elklit A (2015) ICD-11 Trauma Questionnaires for PTSD and Complex PTSD: Validation among Civilians and Former Abducted Children in Northern Uganda. J Psychiatry 18: 335 doi:10.4172/2378-5756.1000335

Page 5 of 9

disorders in the DSM-IV and ICD-10 [17]. M.I.N.I was used to screen for PTSD in $20 \%$ of the participants by random selection. The M.I.N.I was used to help validate the result of the ICD-11 screening measurement. It has previously been used by Abbo et al. [18] to assess psychiatric disorders in northern Uganda, among other places.

\section{Referral}

As per agreement with Victim's Voice (VIVO), participants who fulfilled the criteria for PTSD, by either the ICD-11 or M.I.N.I and who wished to receive help, were referred for counseling at VIVO. A medical doctor from the local health center furthermore agreed to have participants referred.

\section{Data collection and statistical analysis}

Data was collected in the fall of 2014 and analyzed using the SPSS version 22. In SPSS we made frequency analyses, Pearson Correlations, one-way between groups ANOVA tests and Chi-square tests for independence.

\section{Results}

The study found that $97.5 \%$ of the sample was from the Acholi tribe. Of the total sample, $39.5 \%$ had been abducted by the LRA. Both FA (0.7) and NA (0.73) had a mean of less than one owned asset in their household with a mean of two meals a day (Table 1). The total sample had a mean of 3.86 people sleeping in the same room and there was only found a small, but significant difference in the level of education for fathers between FA and NA, for mothers the difference was nonsignificant (Table 1). The age of abduction ranged from 4 years old to 18 years old and the mean age of abduction was 11.4 years $($ median $=12$ years), while the duration of abduction ranged from 1 day to 14 years, with a mean duration of abduction at 1.8 years (SD: 2.4). A Chi-square test for independence indicated no significant association between sex and abduction $\left(\chi^{2}=(1, n=312)=.69, p=.41\right)$. Participants who had been abducted by the LRA had experienced significantly more traumatic events than non-abducted civilians as measured by a one-way betweengroups analysis of variance $(\mathrm{F}=130.48, \mathrm{p}=.0001)$. The three most common traumatic categorical experiences for the total sample were material losses $(96.4 \%)$, separation $(93.6 \%)$, and threats to loved ones (86.4\%). The same ranking was found when looking exclusively at the former abducted individuals (respectively, 99.4\%; 97.6\% and 94.0\%). In addition to the trauma exposure prevalence seen in Table 2, an open question (question $1 \mathrm{~b}$ ) revealed that the most common personal injuries in sorted order were: 'shot', 'wound', 'beaten', 'burn', 'machete cut', 'bomb fragments', 'broken knee', 'leg chopped off' and 'others'. In question 14; "During the war, did you ever see anybody getting confused after hearing about the death of his or her dear ones?" a total of a further $36.9 \%(\mathrm{FA}=41.9 \%$; $\mathrm{NA}=33.9 \%)$ answered yes to having witnessed someone in their household getting confused after hearing about the death of someone.

Nine participants reported that they had left the country during the war in a period ranging from two to ten years. The $54.8 \%$ of FA participants who had received military training had been trained between a week and eight years with a median of 2.0 years. For the category of 'sexual abuse' in the Trauma Exposure Checklist in Table 2 there was only a significant difference between sexes for question 51c. The indications of the Chi-square tests for independence were: 51a: $\chi^{2}=(1, \mathrm{n}=311)=.28,=.60$, phi $=-.04 ; 51 \mathrm{~b}: \chi^{2}=(1, \mathrm{n}=311)=25, \mathrm{p}=.62$, phi $=-.04$; and $51 \mathrm{c}: \chi^{2}=(1, \mathrm{n}=311)=14.99, \mathrm{p}=.0001, \mathrm{phi}=.23$. For the $\mathrm{FA}$ women $65.3 \%$ answered 'yes' to seeing a family member being raped; and $20.2 \%$ answered 'yes' to having been sexual abused themselves. Of the total sample, $36.6 \%$ fulfilled the criteria to be diagnosed with either PTSD or C-PTSD (FA: 46.3\%; NA: 29.7\%) by the ICD-11 screening instrument. There was a significant difference between all PTSD symptoms and the FA and NA, except for one symptom; "Avoiding internal reminders of the stressful experience (for example, thoughts, feelings, or physical sensation)" (Table 3). However, those

Means (SD)

\begin{tabular}{|c|c|c|c|c|}
\hline & $\begin{array}{l}\text { Former } \\
\text { Abducted }\end{array}$ & $\begin{array}{l}\text { Never } \\
\text { Abducted }\end{array}$ & Total Sample & Statistics \\
\hline $\begin{array}{l}1=\text { Intrusive Recollection } \\
\text { Upsetting dreams that replay part of the event or are clearly related to the event? }\end{array}$ & $2.45(1.36)$ & $1.78(1.32)$ & $2.05(1.37)$ & $\mathrm{F}=18.86 \mathrm{P}<.00$ \\
\hline $\begin{array}{l}2 \text { = Distressing Dreams } \\
\text { Powerful images or memories that sometimes come into your mind in which you feel the event is } \\
\text { happening again in the here and now? }\end{array}$ & $2.15(1.39)$ & $1.22(1.32)$ & $1.6(1.42)$ & $\begin{array}{c}F=35.59 \\
P<.00\end{array}$ \\
\hline $\begin{array}{l}3 \text { = Psychological Distress } \\
\text { Feeling very upset when something reminded you of the stressful experience? }\end{array}$ & $2.36(1.26)$ & $1.61(1.16)$ & $1.91(1.26)$ & $\begin{array}{c}\mathrm{F}=28.76 \\
\mathrm{P}<.00\end{array}$ \\
\hline $\begin{array}{l}4 \text { = Avoidance } \\
\text { Avoiding internal reminders of the stressful event experience (for example, thoughts, feelings, or } \\
\text { physical sensations)? }\end{array}$ & $1.67(1.2)$ & $1.2(1.15)$ & $1.39(1.19)$ & $\begin{array}{l}\mathrm{F}=12.11 \\
\mathrm{P}<.00\end{array}$ \\
\hline $\begin{array}{l}5=\text { Avoidance } \\
\text { Avoiding external reminders of the stressful event experience (for example, people, places, } \\
\text { conversations, objects, activities, or situations)? }\end{array}$ & $1.57(1.2)$ & $1.38(1.23)$ & $1.46(1.21)$ & $\begin{array}{c}\mathrm{F}=1.78 \\
\mathrm{~ns}\end{array}$ \\
\hline $\begin{array}{l}6 \text { = Hypervigilance } \\
\text { Being "super-alert", watchful, or on guard? }\end{array}$ & $1.83(1.44)$ & $1.09(1.28)$ & $1.39(1.39)$ & $\begin{array}{c}\mathrm{F}=22.52 \\
\mathrm{P}<.00\end{array}$ \\
\hline $\begin{array}{l}7 \text { = Exaggerated Startle Response } \\
\text { Feeling jumpy or easily startled? }\end{array}$ & $1.77(1.44)$ & $1.27(1.35)$ & $1.48(1.41)$ & $\begin{array}{l}\mathrm{F}=9.85 \\
\mathrm{P}<.00\end{array}$ \\
\hline $\begin{array}{llr}\text { Cluster } & 1 . & \text { Intrusions } \\
\text { Symptom } \geq 2 & & \end{array}$ & $6.96(3.18)$ & $4.63(3.25)$ & $5.57(3.41)$ & $\begin{array}{c}\mathrm{F}=38.99 \\
\mathrm{P}<.00\end{array}$ \\
\hline Cluster 2. Avoidance Symptom $\geq 2$ & $3.25(2.02)$ & $2.58(2.03)$ & $2.86(2.05)$ & $\begin{array}{l}F=8.05 \\
P \leq .01\end{array}$ \\
\hline Cluster 3. Current Threat Symptom $\geq 2$ & $3.62(2.65)$ & $2.36(2.45)$ & $2.87(2.61)$ & $\begin{array}{l}\mathrm{F}=18.33 \\
\mathrm{P}<.00\end{array}$ \\
\hline Total & $13.86(6.26)$ & $9.5(6.2)$ & $11.28(6.58)$ & $\begin{array}{l}F=35.69 \\
P<.00\end{array}$ \\
\hline Positive PTSD \% & 46.3 & 29.7 & 36.6 & \\
\hline
\end{tabular}

Table 3: PTSD as measured by the ICD-11. 
Citation: Dokkedahl S, Oboke H, Ovuga E, Elklit A (2015) ICD-11 Trauma Questionnaires for PTSD and Complex PTSD: Validation among Civilians and Former Abducted Children in Northern Uganda. J Psychiatry 18: 335 doi:10.4172/2378-5756.1000335

Page 6 of 9

who fulfilled the criteria of C-PTSD cannot be diagnosed with PTSD simultaneously. This results in a total of $25.8 \%$ of the total sample receiving a PTSD diagnoses. Thereby, $36.6 \%$ of the total sample suffers from either PTSD or C-PTSD. A Chi-square test for independence indicated no significant association between sex and PTSD $\chi^{2}=(1$, $\mathrm{n}=259)=.79, \mathrm{p}=.38, \mathrm{phi}=.06)$. In 15 of the 17 questions on the C-PTSD diagnostic tool, participants all scored significantly different between FA and NA. The only two questions with a non-significant difference was "Have you suddenly changed your sense of who you are and where you are headed?" and 4: "Does your sense of who you are often change dramatically?". Of the total sample, $14.9 \%$ could be diagnosed with C-PTSD (FA: 24.4\%; NA: 8.1\%). Like PTSD, a Chi-square test for independence indicated no significant association between sex and C-PTSD $\chi^{2}=(1, \mathrm{n}=308)=.86, \mathrm{p}=.35$. As presented in (Table 4), Pearson correlations were used to explore the relationship between the PTSD, C-PTSD-, and APAI-subscale scores. There were medium to high positive correlations among almost all variables except for conduct problems that had small correlations with all variables except for C-PTSD hyperactivation, which had a medium positive correlation with conduct problems. The mean C-PTSD hyperactivation score was 9.87 (Table 5) and the study found that $19.1 \%$ scored above one standard deviation (SD). For C-PTSD deactivation only $8 \%$ scored above one SD and combined $20 \%$ of the participants scoring above one SD for C-PTSD hyperactivation also scored above one SD for C-PTSD deactivation. Prosocial predominantly had non-significant correlations with other variables. Depression/anxiety had high positive significant correlations with all PTSD and C-PTSD variables, except for PTSD avoidance, which had a medium significant positive correlation. A

\begin{tabular}{|c|c|c|c|c|}
\hline \multicolumn{5}{|l|}{ Means (SD) } \\
\hline & $\begin{array}{l}\text { Former } \\
\text { Abducted }\end{array}$ & $\begin{array}{l}\text { Never } \\
\text { Abducted }\end{array}$ & Total Sample & Statistics \\
\hline $\begin{array}{l}1=\text { Hyperactivation } \\
\text { I react intensely to things that don't seem to affect other people so much? }\end{array}$ & $1.81(1.18)$ & $1.48(1.34)$ & $1.61(1.29)$ & $\begin{array}{l}\mathrm{F}=4.84 \\
\mathrm{P} \leq .05\end{array}$ \\
\hline $\begin{array}{l}2 \text { = Hyperactivation } \\
\text { When I am upset, it takes me a long time to calm down. }\end{array}$ & $2.37(1.39)$ & $2.04(1.43)$ & $2.17(1.42)$ & $\begin{array}{l}F=3.94 \\
P \leq .05\end{array}$ \\
\hline $\begin{array}{l}3 \text { = Hyperactivation } \\
\text { My feelings tend to be easily hurt. }\end{array}$ & $2.34(1.45)$ & $2.04(1.53)$ & $2.16(1.50)$ & $\begin{array}{c}\mathrm{F}=2.96 \\
\mathrm{~ns}\end{array}$ \\
\hline $\begin{array}{l}4 \text { = Hyperactivation } \\
\text { I experience episodes of uncontrollable anger. }\end{array}$ & $2.33(1.42)$ & $2.04(1.44)$ & $2.15(1.43)$ & $\begin{array}{c}\mathrm{F}=2.92 \\
\mathrm{~ns}\end{array}$ \\
\hline $\begin{array}{l}5=\text { Hyperactivation } \\
\text { I do things that people have told me are dangerous or reckless (for example, driving very fast). }\end{array}$ & $2.2(1.18)$ & $1.88(1.35)$ & $2.01(1.29)$ & $\begin{array}{l}\mathrm{F}=4.32 \\
\mathrm{P} \leq .05\end{array}$ \\
\hline $\begin{array}{l}6=\text { Deactivation } \\
\text { I feel numb or emotionally shut down. }\end{array}$ & $1.12(1.17)$ & $.68(.99)$ & $.86(1.09)$ & $\begin{array}{l}F=12.22 \\
P \leq .001\end{array}$ \\
\hline $\begin{array}{l}7 \text { = Deactivation } \\
\text { I am the kind of person who has difficulty experiencing feelings of pleasure or joy. }\end{array}$ & $1.93(1.35)$ & $1.52(1.28)$ & $1.68(1.32)$ & $\begin{array}{l}F=6.96 \\
P \leq .01\end{array}$ \\
\hline $\begin{array}{l}8 \text { = Deactivation } \\
\text { When I am under stress or confronted with reminders of my trauma, I often feel that the world is distant or } \\
\text { that the world seems different (for example, time slows down, things look different) }\end{array}$ & $2.29(1.33)$ & $1.64(1.25)$ & $1.91(1.33)$ & $\begin{array}{c}F=18.73 \\
P<.00\end{array}$ \\
\hline $\begin{array}{l}9 \text { = Deactivation } \\
\text { When I am under stress or confronted with reminders of my trauma, I often feel outside my body or feel that } \\
\text { there is something strange about my body }\end{array}$ & $2.07(1.22)$ & $1.54(1.21)$ & $1.76(1.24)$ & $\begin{array}{c}F=14.36 \\
P \leq .00\end{array}$ \\
\hline $\begin{array}{l}10=\text { Deactivation } \\
\text { I have difficulty knowing what I feel and describing my feelings. }\end{array}$ & $1.88(1.52)$ & $1.21(1.37)$ & $1.47(1.47)$ & $\begin{array}{l}\mathrm{F}=16.26 \\
\mathrm{P} \leq .00\end{array}$ \\
\hline $\begin{array}{l}11=\text { Negative Self-Concept } \\
\text { I feel like a failure. }\end{array}$ & $2.09(1.32)$ & $1.51(1.29)$ & $1.75(1.33)$ & $\begin{array}{l}\mathrm{F}=14.51 \\
\mathrm{P}<.00\end{array}$ \\
\hline $\begin{array}{l}12=\text { Negative Self-Concept } \\
\text { I feel worthless. }\end{array}$ & $2.03(1.40)$ & $1.33(1.34)$ & $1.61(1.41)$ & $\begin{array}{l}\mathrm{F}=19.05 \\
\mathrm{P} \leq .00\end{array}$ \\
\hline $\begin{array}{l}13=\text { Negative Self-Concept } \\
\text { I often feel ashamed of myself whether it makes sense or not. }\end{array}$ & $1.83(1.27)$ & $1.30(1.29)$ & $1.45(1.32)$ & $\begin{array}{l}\mathrm{F}=17.52 \\
\mathrm{P} \leq .00\end{array}$ \\
\hline $\begin{array}{l}14=\text { Negative Self-Concept } \\
\text { I feel guilty about things I have done or failed to do. }\end{array}$ & $2.24(1.25)$ & $1.69(1.36)$ & $1.92(1.34)$ & $\begin{array}{l}\mathrm{F}=12.69 \\
\mathrm{P} \leq .00\end{array}$ \\
\hline $\begin{array}{l}15 \text { = Disturbed Relationship } \\
\text { I feel distant or cut off from people. }\end{array}$ & $2.13(1.38)$ & $1.47(1.32)$ & $1.74(1.39)$ & $\begin{array}{l}\mathrm{F}=7.93 \\
\mathrm{P} \leq .00\end{array}$ \\
\hline $\begin{array}{l}16=\text { Disturbed Relationship } \\
\text { I find it hard to stay emotionally close to people. }\end{array}$ & $1.77(1.39)$ & $1.08(1.26)$ & $1.36(1.36)$ & $\begin{array}{l}F=21.08 \\
P \leq .00\end{array}$ \\
\hline $\begin{array}{l}17=\text { Disturbed Relationship } \\
\text { I avoid relationships because they end up being too difficult or painful. }\end{array}$ & $1.44(1.39)$ & $.96(1.23)$ & $1.16(1.32)$ & $\begin{array}{l}F=9.96 \\
P \leq .002\end{array}$ \\
\hline $\begin{array}{llll}\text { Items } & 1-5 & \text { sum } & \text { score } \\
\text { Hyperactivation } & & \end{array}$ & $10.71(4.40)$ & $9.37(4.98)$ & $9.87(4.80)$ & $\begin{array}{l}\mathrm{F}=5.14 \\
\mathrm{P} \leq .03\end{array}$ \\
\hline $\begin{array}{l}\text { Items } 6-10 \text { sum score }(\geq 8) \\
\text { Deactivation }\end{array}$ & $9.42(4.52)$ & $6.62(4.26)$ & $7.75(4.57)$ & $\begin{array}{l}\mathrm{F}=27.94 \\
\mathrm{P} \leq .00\end{array}$ \\
\hline $\begin{array}{l}\text { Items } 10-14 \text { sum score }(\geq 10) \\
\text { Negative Self-Concept }\end{array}$ & $8.01(4.36)$ & $5.67(4.13)$ & $6.60(4.37)$ & $\begin{array}{l}\mathrm{F}=21.70 \\
\mathrm{P} \leq .00\end{array}$ \\
\hline $\begin{array}{l}\text { Items } 15-17 \text { sum score }(\geq 6) \\
\text { Disturbed Relationships }\end{array}$ & $5.33(3.47)$ & $3.51(3.16)$ & $4.26(3.43)$ & $\begin{array}{l}\mathrm{F}=22.72 \\
\mathrm{P} \leq .00\end{array}$ \\
\hline Total & $33.03(13.80)$ & $24.58(13.82)$ & $27.67(14.37)$ & $\begin{array}{l}\mathrm{F}=21.62 \\
\mathrm{P} \leq .00\end{array}$ \\
\hline Positive C-PTSD \% & 24.4 & 8.1 & 14.9 & \\
\hline
\end{tabular}

Table 4: C-PTSD as measured by the ICD-11. 
one-way between-groups analysis of variance was conducted to explore the impact of 11 types of trauma events on PTSD, C-PTSD and the APAI as seen in (Table 6). Predominantly PTSD, C-PTSD, depression/ anxiety and somatic complaints were statistically significant different from the 11 traumatic events at the $\mathrm{p}<.05$ level with the exception of material losses, displacement and separation. Threats to loved ones was non-significant for somatic complaints and threats to your loved ones was non-significant for C-PTSD. Conduct problems and prosocial was not associated with the 11 types of trauma events in the one-way between-groups analysis of variance except for sexual abuse, which was significant at the $\mathrm{p}<.05$ level for prosocial. Comparing the PTSD results, a Chi-square test for independence indicated no significant association between PTSD as measured by the ICD-11 tools and PTSD measured by the M.I.N.I $\chi^{2}=(1, \mathrm{n}=61)=1.63, \mathrm{p}=.2$, $\mathrm{phi}=.2$. Using the Kappa Measure of Agreement the sensitivity and specificity between M.I.N.I and ICD11 was calculated. The Kappa Measure of Agreement value was .2 and was non-significant with $\mathrm{p}=.12$. Therefore, level of agreement between PTSD as measured by ICD-11 and PTSD as measured by M.I.N.I is small and non-significant. Of the 27 participants identified with PTSD by the M.I.N.I, 14 were also calculated with PTSD on the ICD-11. This represents a sensitivity value of $51.9 \%$. Of the 34 identified as not having PTSD by the M.I.N.I, 23 were also calculated as not having PTSD by the ICD-11. This represents a specificity rate of $67.7 \%$.

\section{Discussion}

When renewing a diagnostic system like the ICD-11 it is important to consider its clinical utility in different clinical settings, including developing countries like Uganda. In addition, validating the diagnostic tools is equally important before implementing a new diagnosis. The aim of the current paper is to discuss the utility of the PTSD and C-PTSD diagnoses in the ICD-11 as well as validating the proposed measurement tools, respectively.

As previously mentioned, C-PTSD arises after the "exposure to a stressor typically of an extreme or prolonged nature and from which escape is difficult or impossible" [4]. As expected, many of both the FA and the NA of the Awach community seemed to have been exposed to such traumas. Many had been abducted by the LRA, and the two most common traumatic experiences were 'material losses' and 'separation'. Likewise, 'threats to loved ones', 'injuries and threats to self or loved ones', and 'witnessing violence' also had high prevalence. Interestingly, neither 'material losses' nor 'separation' appears to have many psychological consequences as measured by ICD and APAI (Table 7). Opposite, 'threats to loved ones', 'injuries and threats to self or loved ones', and 'witnessing violence' are all associated with PTSD, C-PTSD, Depression/Anxiety and Somatic Complaints for 'injuries and threats to self or loved ones' and 'witnessing violence' (Table 6). The difference is possibly explained by the circumstances of the war, where separation and material losses were common (Table 2) and therefore expected. Sexual abuse had a relatively low prevalence compared to knowledge about abducted girls [12]. This might be explained by the cultural stigmatization associated with rape, which could have lead to a underreporting of sexual abuse. Compared to former studies in northern Uganda, the prevalence of PTSD seemed to be relatively low with $25.8 \%$ fulfilling the PTSD diagnosis for ICD-11 in the total sample ( $\mathrm{FA}=30.4 \%$; $\mathrm{NA}=23.2 \%)$. However, if the C-PTSD diagnosis fulfilled by $14.9 \%$ were included, the total prevalence of PTSD and C-PTSD would be $36.6 \%(\mathrm{FA}=46.3 \%$; NA=29.7\%) of the total sample, which is in accordance with the findings of PTSD in former studies. It can be speculated that the previous diagnostic tools would have captured both groups of PTSD and C-PTSD under one PTSD diagnosis.
Several different PTSD diagnostic tools have been used; among them the Harvard Trauma Questionnaire and the Posttraumatic-Stress Diagnostic Scale were some of the most commonly used. As expected, the FA had higher prevalence of C-PTSD, as well as PTSD, compared to the NA, which is in accordance with higher numbers of traumatic events experienced by the FA. This suggests discriminant validation of the ICD-11 measurement tools for PTSD and C-PTSD and supports their clinical utility in developmental settings. The results showed no significant association between the findings of the M.I.N.I PTSD and the ICD-11 PTSD diagnosis. In addition, the sensitivity and specificity was relatively low. A possible explanation for the relatively low sensitivity is the necessity of responding to the traumatic experience with 'intense fear, helplessness or horror' for a M.I.N.I diagnosis of PTSD. This distinguishes the M.I.N.I from the ICD-11, seeing that this criterion has not been included by the WHO Working Group. The current study found that $17.6 \%$ of the participant answering 'yes' to having experience a traumatic event in the M.I.N.I answered 'no' to having responded to it with fear, helplessness or horror. This study further found that $44.4 \%$ of those answering 'no' to having responded to the trauma with fear, helplessness or horror in the M.I.N.I, were diagnosed with PTSD by the ICD-11. Future research can benefit from studying this issue in a bigger sample. The war in northern Uganda lasted for two decades during which traumatic events were common. Those who have not been exposed to abduction or violence themselves, are likely to have witnessed it on someone they knew or having experienced living in IDP camps. It can be speculated that constant exposure to traumatic events might have led to a psychological state of which an actual trauma is no longer experienced with fear, helplessness or horror for the people of northern Uganda. Future research may also benefit from this speculation. PTSD is known for having a high comorbidity with other mental health problems like depression and anxiety [8]. The current study made similar findings with high positive correlations between PTSD/C-PTSD and symptoms of depression/ anxiety as measured by the APAI. Likewise, there are small but significant positive correlations with conduct problems and somatic complaints. The positive correlations suggest convergent validation for the measures of the ICD-11 tools for PTSD and C-PTSD seeing that these are in accordance with the knowledge on comorbidity of PTSD [8]. The statements in the APAI that regards conduct problems are in accordance with some of the key features of C-PTSD. The APAI asks about substance abuse, insults of friends, fights, being a rough person and being disobedient, among others. Similarly, C-PTSD symptoms of emotional dysregulation and interpersonal difficulties are observed on adolescents through substance abuse, risky behaviors and aggressive behaviors [4]. In Table 5 it is apparent that for conduct problems the highest correlation is C-PTSD hyperactivation, but C-PTSD deactivation also has a relatively high correlation with conduct problems (compared with other correlations for conduct problems; Table 5). With $20 \%$ of the participants scoring positive for hyperactivation also scoring positive for deactivation it indicates that conduct problems can be both externalizing as well as internalizing. However, from Table 6 it is evident that conduct problems and prosocial behavior is not linked to any of the traumatic exposures. This indicates that conduct problems and prosocial behavior are personality traits, which are not triggered by any specific traumatic event. Furthermore, somatic complaints are not key features of the ICD-11 PTSD diagnosis due to the fact that somatic complaints can occur without the presence of an external stressor. In addition to war trauma events, a possible trigger for both somatic complaints and conduct problems could be the extreme poverty that people in northern Uganda live in. In Table 1 it is evident that the mean number of assets in each household is 
Citation: Dokkedahl S, Oboke H, Ovuga E, Elklit A (2015) ICD-11 Trauma Questionnaires for PTSD and Complex PTSD: Validation among Civilians and Former Abducted Children in Northern Uganda. J Psychiatry 18: 335 doi:10.4172/2378-5756.1000335

Page 8 of 9

\begin{tabular}{|c|c|c|c|c|c|c|c|c|c|c|c|}
\hline & 1. & 2. & 3. & 4. & 5. & 6. & 7. & 8. & 9. & 10. & 11. \\
\hline 1. PTSDsum1 $=$ Intrusions & 1 & $.49^{\circ}$ & $.63^{*}$ & $.50^{*}$ & $.64^{*}$ & $.59^{*}$ & $.56^{*}$ & $.61^{*}$ & $.12^{\star \star}$ & $.40^{*}$ & .02 \\
\hline 2. PTSDsum2 = Avoidance & & 1. & $.26^{*}$ & $.31^{*}$ & $.42^{*}$ & $.26^{*}$ & $.31^{*}$ & $.32^{*}$ & .10 & $.30^{*}$ & $-.17^{\star}$ \\
\hline 3. PTSDsum3 = Current Threat & & & 1. & $.37^{*}$ & $.62^{*}$ & $.60^{*}$ & $.57^{*}$ & $.55^{*}$ & $.14^{*}$ & $.26^{*}$ & $.20^{*}$ \\
\hline 4. C-PTSD Hyperactivation & & & & 1. & $.57^{*}$ & $.59^{*}$ & $.45^{*}$ & $.53^{*}$ & $.34^{*}$ & $.46^{*}$ & .01 \\
\hline 5. C-PTSD Deactivation & & & & & 1. & $.75^{*}$ & $.75^{*}$ & $.66^{*}$ & $.26^{*}$ & $.44^{*}$ & .06 \\
\hline 6. C-PTSD Negative Self-Concept & & & & & & 1. & $.71^{*}$ & $.68^{*}$ & $.22^{*}$ & $.44^{*}$ & $.12^{* *}$ \\
\hline 7. C-PTSD Disturbed Relationships & & & & & & & 1. & $.62^{*}$ & $.19^{*}$ & $.37^{*}$ & .07 \\
\hline 8. Depression/Anxiety & & & & & & & & 1. & $.28^{*}$ & $.68^{*}$ & $.21^{*}$ \\
\hline 9. Conduct Problems & & & & & & & & & 1. & $.24^{*}$ & $.14^{*}$ \\
\hline 10. Somatic Complaints & & & & & & & & & & 1. & -.04 \\
\hline 11. Prosocial & & & & & & & & & & & 1. \\
\hline
\end{tabular}

Sig. (2-tailed). ${ }^{*}=$ correlation is significant at the 0.01 level ${ }^{* *}=$ correlation is significant at the 0.05 level.

Table 5: Pearson correlations between PTSD, C-PTSD \& APAI.

\begin{tabular}{|c|c|c|c|c|c|c|}
\hline Trauma events (11) & PTSD & CPTSD & Depression Anxiety & Conduct Problems & Somatic Complaints & Prosocial \\
\hline A. Personal Injury & $F=2.98^{*}$ & $\mathrm{~F}=2.51^{*}$ & $F=2.18^{*}$ & $F=.80$ & $F=4.93^{*}$ & $F=1.50$ \\
\hline B. Witnessing Violence & $F=2.85^{*}$ & $F=1.54^{* *}$ & $F=1.86^{*}$ & $\mathrm{~F}=.53$ & $F=3.51^{*}$ & $F=1.26$ \\
\hline C. Injuries and Threats to Loved Ones & $F=3.24^{*}$ & $1.56^{* *}$ & $F=2.17^{*}$ & $F=.67$ & $F=6.69^{*}$ & $F=1.39$ \\
\hline D. Deaths & $F=2.88^{*}$ & $F=1.84$ * & $F=2.10^{*}$ & $F=1.07$ & $F=5.24^{*}$ & $F=1.08$ \\
\hline E. Threaths to Loved Ones & 2.79 * & $F=1.66^{*}$ & $F=1.81^{*}$ & $\mathrm{~F}=.53$ & $F=1.59$ & $F=1.27$ \\
\hline F. Material Losses & $F=1.39$ & $F=.72$ & $F=0.88$ & $F=.40$ & $F=1.11$ & $F=1.66$ \\
\hline G. Threats to Your Loved Ones & $\mathrm{F}=2.96$ * & $F=1.34$ & $F=1.70^{*}$ & $F=.75$ & $\mathrm{~F}=3.75$ * & $F=1.16$ \\
\hline H. Separation & $F=.64$ & $F=.96$ & $F=.97$ & $F=1.28$ & $F=2.09^{* *}$ & $F=.44$ \\
\hline I. Displacement & $F=.88$ & $F=.73$ & $F=1.05$ & $F=1.19$ & $F=1.82$ & $F=1.16$ \\
\hline J. Participating in Armed Groups & $\mathrm{F}=1.67^{* *}$ & $F=1.71^{*}$ & $\mathrm{~F}=1.52^{* *}$ & $F=.54$ & $F=1.70$ & $F=1.35$ \\
\hline K. Sexual Abuse & $F=3.43^{*}$ & $F=1.59^{*}$ & $F=1.70^{*}$ & $F=.69$ & $F=3.13^{*}$ & $\mathrm{~F}=2.07^{* *}$ \\
\hline
\end{tabular}

${ }^{*}=p<.01 .{ }^{* *}=p<.05$

Table 6: Comparing means for mental health and traumatic events.

\begin{tabular}{|c|c|c|}
\hline \multicolumn{3}{|c|}{ ICD-11 and M.I.N.I (N =62) } \\
\hline & ICD-11 = YES & ICD 11 = NO \\
\hline M.I.N.I = YES & 14 & 13 \\
\hline M.I.N.I = NO & 11 & 23 \\
\hline
\end{tabular}

Table 7: Comparing PTSD by ICD-11 and M.I.N.I.

less than one and very few people are able to get education extending from primary school. However, somatic complaints are common complaints among people suffering from PTSD [13,14]. Therefore, the presence of somatic complaints helps support the ICD-11 diagnostic tools through positive correlations with PTSD and C-PTSD. Like for somatic complaints, the Trauma Exposure Checklist asked participants about experiencing a feeling of confusion (question 14). Confusion is not a symptom of PTSD and C-PTSD in the ICD-11 [15]. However, confusion can be said to be a phenomenological condition for people with PTSD similar to somatic complaints. As stated by Maercker et al. [4] the ICD-11 PTSD diagnosis is supposed to focus only on the core elements of PTSD. The seven components of the diagnostic tool therefore ask only about intrusions, avoidance and hypervigiliance. The diagnosis was made this way to help increase clinical utility. The findings from this study indicate that the new edition of the ICD
PTSD diagnosis helps provide a more clear definition of the elements of the diagnosis of PTSD. By providing the opportunity of a C-PTSD diagnosis, some patients who would previously have received a PTSD diagnosis now receive a diagnosis of C-PTSD allowing the clinicians to focus on symptoms, problems, and the target of treatment [7], which could benefit the outcome for the patient.

\section{Limitations}

A limitation to this study was the lack of proper random sampling and the selection of participants, which due to the lack of a national register in Uganda instead was selected by the LC1s, based on the ideas of random sampling. In addition, the instruments translated into the local language Luo has not been validated. Furthermore, the seven years that has passed since the war ended may contribute to some recall bias. However, the traumatic experiences during the war were severe enough to fulfill the 'stress-criteria' for C-PTSD. The interviews were conducted in people's homes, which might have led to some inevitable disturbances although quiet places were sought out and field assistants allowed as much time for the interviews as needed. 
Citation: Dokkedahl S, Oboke H, Ovuga E, Elklit A (2015) ICD-11 Trauma Questionnaires for PTSD and Complex PTSD: Validation among Civilians and Former Abducted Children in Northern Uganda. J Psychiatry 18: 335 doi:10.4172/2378-5756.1000335

Page 9 of 9

\section{Further Studies}

The current study found that certain symptoms of PTSD and C-PTSD were more common than others. For PTSD the least common symptom was avoidance while the least common symptom for C-PTSD was disturbed relationships (Table 6). Future studies may look into these differences to find out whether they represent a common distribution for PTSD and C-PTSD respectively, or if they are influenced by cultural differences. Uganda is a collectivistic society, which may have an influence on people's perception on the symptoms of disturbed relationships. People in Uganda rely on their families and marriage is often decided as an agreement between families. Children are further a financial security for the future and few people have the luxury of getting a good education $[19,20]$. The mere concept of disturbed relationships might be misunderstood in a collectivistic society, seeing that few people have the choice of withdrawing from other people. Marriage is often a practical decision and may not be comparative to the emotional bond we associate it with in individualistic cultures. Likewise, symptoms of emotional distance and avoiding relationships could be less relevant in a culture that view emotional expression as taboo. Furthermore, in a society where everybody is likely to have been exposed to similar traumas, the idea of social withdrawal might be less relevant. Future research could benefit from comparing the distribution of symptoms between cultures. Compared to universal findings on the prevalence of PTSD [21], the prevalence of PTSD in this study appears high. But when comparing the prevalence of PTSD to the extent of the trauma exposure that the participants in this study have experienced, the prevalence of PTSD and C-PTSD may actually be quite low. Stein et al. [22] have argued that too much focus on illness can influence a population who are in fact well, but also well-informed about disease, to complain more about disorders, compared to uninformed populations. In this sense, people in Uganda would be less likely to complain about their symptoms due to the lack of knowledge they have about the concept of mental ill health. Again, future research can benefit from this speculation.

\section{Conclusion}

The findings of this study support the previous findings of studies on C-PTSD $[7,11]$. This indicates that the diagnostic suggestions of the ICD-11 are suited to describe the consequences of traumatic experiences in a developmental country like Uganda. As expected, the traumatic experiences during the war in northern Uganda had high associations with PTSD and C-PTSD, and C-PTSD seems to be associated with other psychological problems like depression, anxiety, and somatic complaints. The current study further concludes that the ICD-11 tools for PTSD and C-PTSD both appear to be valid as suggested by both discriminant and convergent validation of the tools.

\section{References}

1. Herman JL (1992) Complex PTSD: A Syndrome in Survivors of Prolonged and Repeated Trauma. J Traumatic Stress 5: 377-391.

2. Evans SC, Reed GM, Roberts MC, Esparza P, Watts AD, et al. (2013) Psychologists' perspective in the diagnostic classification of mental disorders: Results from the WHO-IUPsyS Global Survey. Int J Psychol 48: 177-193.

3. Reed GM, Correia JM, Esparza P, Saxena S, Maj M (2011) The WPA-
WHO Global Survey of Psychiatrists' Attitudes Towards Mental Disorders Classification. World Psychiatry 10: 118-131.

4. Maercker A, Brewin CR, Bryant RA, Cloitre M, van Ommeren M, et al. (2013) Diagnosis and classification of disorders specifically associated with stress: proposal for ICD-11. World Psychiatry 12: 198-206.

5. 5. Maercker A, Brewin CR, Bryant RA, Cloitre M, Reed GM, et al. (2013) Proposal for mental disorders specifically associated with stress in the International Classification of Diseases-11. The Lancet 381: 1683-1685.

6. Friedman MJ (2013) Finalizing PTSD in DSM-5: Getting Here From There and Where to Go Next. J Traumatic Stress 26: 548-556.

7. Cloitre M, Garvert DW, Brewin CR, Bryant RA, Maercker A (2013) Evidence for proposed ICD-11 PTSD and complex PTSD: a latent profile analysis. European J Psychotraumatol 4: 20706.

8. Simms LJ, Watson D, Doebbeling BN (2002) Confirmatory Factor Analyses of Posttraumatic Stress Symptoms in Deployed and Nondeployed Veterans of the Gulf War. J Abnormal Psychol 111: 637-647.

9. Brewin CR, Lanius RA, Novac A, Schnyder U, Galea S (2009) Reformulating PTSD for DSM.V: Life after Criterion A. J Traumatic Stress 22: 366-373.

10. Peters L, Slade T, Andrews G (1999) A Comparison of ICD10 and DSM-IV Criteria for Posttraumatic Stress Disorder. J Traumatic Stress 12: 335-343.

11. Elklit A, Hyland P, Shevlin M (2014) Evidence of symptom profiles consistent with posttraumatic stress disorder and complex posttraumatic stress disorder in different trauma samples. European J Psychotraumatology 5: 24221.

12. Child Soldiers Global Report (2008) Retrieved on October $7^{\text {th }} 2014$

13. Pfeiffer A, Elbert T (2011) PTSD, depression and anxiety among former abductees in Northern Uganda. BMC Conflict and Health 5.

14. Amone-P'Olak K, Jones PB, Abbott R, Meiser-Stedman R, Ovuga E, et al. (2013) Cohort profile: mental health following extreme trauma in a northern Uganda cohort of War-Affected Youth Study (the WAYS Study). Springer Plus 2.

15. Cloitre Roberts, Bisson, Brewin (2013) Self-Report Community Version 1.0 September 122013.

16. UNICEF (2010) B\&H Postwar Screening Survey.

17. Sheenan DV, Lecrubier E (1992-2006) M.I.N.I. Mini International Neuropsychiatric Interviews. 5.0.0.

18. Abbo C, Kinyanda E, Kizza RB, Levin J, Ndyanabangi S, et al. (2013) Prevalence, comorbidity and predictors of anxiety disorders in children and adolescents in rural north-eastern Uganda. Child and Adolescent Psychiatry and Mental Health 7 .

19. Pallant J (2010) SPSS Survival Manual. Open University Press. McGraw-Hil Education: New York.

20. Mental Health and Peacebuilding in Acholiland. The United Nations Peacebuilding Programme in Uganda, 2011.

21. Norris FH, Sloane LB (2007) The Epidemiology of Trauma and PTSD. In Friedman MJ, Keane TM, Resick PA (Eds.): Handbook of PTSD.

22. Stein DJ, Seedat S, Iversen A, Wessely S (2007) Post-traumatic stress disorder: medicine and politics. The Lancet 369: 139-144. 